\title{
EMBEDDING BLENDED LEARNING ENVIRONMENTS IN HIGHER EDUCATION: TOWARDS A EUROPEAN MATURITY MODEL
}

Katie Goeman, Stephan Poelmans, Veerle Van Rompaey, KU Leuven, Belgium, Wiebe Dijkstra, Willem Van Valkenburg, TU Delft, The Netherlands

\section{The EMBED Project: A Quest for a European Maturity Model}

\section{A Partnership in Higher Education}

The "European Maturity Model for Blended Education" (EMBED) is a higher education (HE) project (2017-2020) led by a consortium of universities across Europe: KU Leuven, TU Delft, the University of Edinburgh, the University of Aarhus, Dublin City University and Tampere University of Applied Sciences. It is coordinated by The European Association of Distance Teaching Universities (EADTU) and funded by the European Commission.

Aim is to support institutions when introducing, developing and implementing blended learning (BL) in HE. A reference model is created that encompasses all levels of an institution: the design of a course, organisational aspects such as policy making, staff support, training and leadership, while guarding the institution's quality culture. Internal stakeholders include learners, teaching staff, support services, technology departments and university leaders, while external stakeholders are key persons responsible for policy making such as governments, European university networks and the EU.

\section{Basic Assumptions of the Maturity Model}

The European maturity model or EMM includes criteria and instruments to assess the degree of maturity of BL in a $\mathrm{HE}$ institution. It is conceived as a staged maturity model; it includes a framework for change, based on progress markers related to stakeholder-focused outcomes. To this end, the aim is to look at the tangible practices at the micro level (courses and programme) and the meso-level (strategies and conditions) in place at a particular institution. It focuses on practices and conditions in-place, not processes nor input-output procedures. In accordance with its logic, maturity of a practice indicates a more holistic approach in terms of design, informed by evidence or experience. Maturity does not equal better practice, maturity equals in this view being "more comprehensive". In each of the maturity levels QA standards and indicators can assess the quality of a particular practice. However, this is not the aim of the EMM. The EMM is designed to assess blended teaching and blended education in terms of different levels of maturity, and to propose follow-up actions. These actions are embedded in the particular context institutions operate in. 


\section{The Project Phases}

The project partners embrace a multilevel framework in order to tackle conceptual and implementation issues at the course level (micro), at the strategic level (meso) and with the intent to give relevant input to governmental policy (macro). Figure 1 depicts the different phases of the EMBED project:

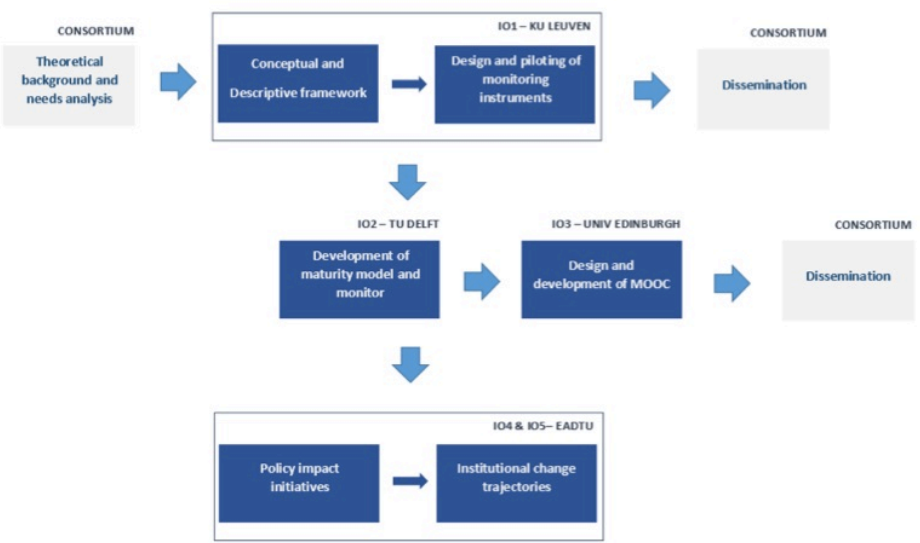

Figure 1. Phases of the EMBED Project

\section{The EMBED Project: Status of Affairs}

Between November and December 2018, TU Delft and KU Leuven have guided the transition from iO1 to iO2, and currently (February 2019) the design and development of a MOOC is being prepared. In the following paragraph the prominent past activities are described.

\section{Conceptual and Descriptive Framework}

During $\mathrm{iO}$ a thorough literature review was carried out to get an overview on current conceptions, theories and models. The desk research resulted in a general conceptual and operational framework, the blueprint of EMBED. It includes the outline of key terminology principles on blended learning, teaching and education (see also Goeman, Poelmans, \& Van Rompaey, 2018). It allows researchers, practitioners and policymakers to talk common language and design, develop and assess current practices, strategical and implementation conditions in a systematic manner.

BL was defined as "learning as a result of a deliberate, integrated combination of online and face-to-face learning activities", while blended teaching (BT) was described as "designing and facilitating blended learning activities". The term deliberate refers to the explicit role of a design which specifies the proportion and sequencing of online and offline learning activities (course) or courses (program). The term 'integrated combination' refers to the logic for using a particular "blend" of virtual and physical spaces. Finally, blended education (BE) refers to "the formal context of $\mathrm{BL}$ (practices) that is determined by policies and conditions with regard to the organization and support of blended learning”. It impacts hybrid (re)designs of courses and programs by the nature of their policies and conditions for organizing and supporting BL and BT. The complete process encompasses 4 iterative phases with particular ground(s) of decision guiding these phases (Table 1): 
Table 1: $\quad$ The ground(s) of decisions and design of BL practices

\begin{tabular}{|c|c|c|}
\hline & Design ground & Design phase \\
\hline 1 & $\begin{array}{l}\text { Context and curriculum } \\
\text { specifications }\end{array}$ & $\begin{array}{l}\text { Set up the blended learning environment } \\
\text { What is to be learned? - define learning objectives and contents } \\
\text { at course and program level } \\
\text { Who is involved? (learner and instructor characteristics) - define } \\
\text { the attributes of the LE } \\
\text { What resources are available? - assess the implementation and } \\
\text { change conditions that shape the teaching and learning context }\end{array}$ \\
\hline 2 & $\begin{array}{l}\text { Learner, program and } \\
\text { instructor profile }\end{array}$ & $\begin{array}{l}\text { Determine flexibility at course and program level: } \\
\text { To what extent is flexibility offered? - determine one or more } \\
\text { categories and dimensions of flexibility }\end{array}$ \\
\hline 3 & $\begin{array}{l}\text { Learning theory / } \\
\text { Instructional design } \\
\text { model }\end{array}$ & $\begin{array}{l}\text { Define learning activities and their sequencing in line with learning } \\
\text { objectives: } \\
\text { Which learning tasks are offered, composed by the didactical } \\
\text { components content, communication and construction and what } \\
\text { is their grouping (individual vs group)? } \\
\text { What learning activities will take place online, which onsite, in } \\
\text { what order? }\end{array}$ \\
\hline 4 & $\begin{array}{l}\text { Learning activities and } \\
\text { media affordances }\end{array}$ & $\begin{array}{l}\text { Choose tools for delivery and organization of the learning activities: } \\
\text { Which tools are employed? - determine interactivity and } \\
\text { synchronicity as a function of media affordances }\end{array}$ \\
\hline
\end{tabular}

\section{Design and Piloting of Monitoring Instruments}

In parallel to the desk research, instrumentation for multilevel monitoring purposes was designed and piloted. Objective was to understand in detail which BL environments are in place staff, which designs for BL designers and teaching staff in higher education adhere to (and why), and the way these practices are reinforced by the university context, i.e. the strategical measures and operational conditions. Moreover, it tries to explain which factors promote or hamper maturity transition, at the course, programme and/or institutional level.

A mixed-method field study was set up across the different partner universities. This encompassed an online questionnaire and semi-structured interviews. A range of knowledge domains were covered, such as ICT, Nursing, Teacher Education, History, Economics. Detailed quantitative data was collected regarding the number and nature of both online and offline learning activities, next to tools for delivery and organisation of blended courses. The findings show that the flipped classroom approach is very popular as a BL design. The most frequently used resources, media and tools are not sophisticated. Detailed qualitative data was collected during in-depth interviews with policy makers, support and teaching staff. To this end, a protocol was developed which was translated in different languages. Many cases are driven by the lecturers' personal interest, their wish to improve the quality of in-class time, and to encourage deeper learning. The respondents' perceived advantages of BT include: active learning, strengthening of learning experiences, enhanced interactivity, and more contemporary education. Difficulties and crucial success factors were also identified. The analysis of the qualitative data suggests the following core themes: added value and constraints of $\mathrm{BL}$ approaches, drivers for BL implementation, course interaction, course 
design, course sustainability, institutional view on BL and institutional support, propagation of practices.

\section{Development and Review of the Maturity Model}

With the overarching themes and critical issues being identified, the next step involved the definition of different levels, dimensions and indicators of maturity. For this purpose, a preDelphi study and an expert panel were conducted between December 2018 and January 2019. The pre-Delphi study's objective was to assess the wording (labelling, clear understanding) of the dimensions of the EMM, and its completeness (definitions, demarcations of dimensions and levels of maturity). During the MID2018 conference in Maastricht, subgroups of participants generated feedback on the interim model. Their input, along with the outcomes of the pre-Delphi study has led to an enhanced version of the EMM.

The current maturity model consists of three maturity levels, subdivided in dimensions and indicators considered crucial for discerning course and programme practices, next to institutional conditions and strategies. These were deducted from literature and the series of research, development and review activities as previously described.

\section{Dissemination Activities}

Throughout the whole project dissemination activities, including multiplier events and training initiatives, are organised on a regular basis. The purpose is to create awareness about the EMBED project, its progress and results (see https://embed.eadtu.eu). It gives different (local) stakeholders (students, teaching and support staff, course designers and programme coordinators, university leaders) the opportunity to learn about the core insights of the project, and benefit from the project instruments for practice and policy making. In September 2018, for example, a 3-day training was organized at the Tampere University of Applied Sciences. Training activities were clustered around the following topics: (a) General framework - Blended learning, teaching and education, (bc Blended learning design, (c) Embedding existing materials in a BL environment, (d) Embedding interactivity and tools in a BL environment and (e) Embedding video and screencast in a BL environment.

\section{The EMBED Project: An Outlook and a Critical Retrospect}

TU Delft will establish the final version of the EMM based on the outcomes of a 3-round Delphi study. The Delphi method allows to collect and interpret a collective point of view of expert-participants in order to generate empirical validation. An international panel of experts is invited to critically review in a grounded way the complete EMM. Throughout three rounds they will receive questionnaires which constitute Likert-scale assessments of each indicator per action level, ranked and described in detail per maturity level. The expert feedback, including convergent and divergent views, is analysed statistically and incorporated after each round (between round 1 and 2, between round 2 and 3). The results give impetus to shape the questionnaire in the subsequent round. 
The strength of the EMBED project is at the same time its Achille's heel. Even though a profound literature review was carried out which allowed to assess recent evidence on BL and related topics and terms, it is still difficult to reach consensus within the project consortium on the terminology and, consequently, operational procedures and instruments. In this regard, using the Delphi method to define each of the general EMBED parts, as well as assess the completeness and appropriateness of the specific dimensions, indicators and criteria is an asset. The diversified composition of the expert panel allows to consider different perspectives and experiences, and to work towards an agreed multilevel, multidimensional model.

\section{References}

Goeman, K., Poelmans, S., \& Van Rompaey, V. (2018). Framing Blended Learning, Teaching and Education. ICERI2018 Proceedings, Sevilla, 1676-1680.

EMBED (2017). European Maturity model for Blended Education. Retrieved from https://embed.eadtu.eu/en 